Check for updates

Cite this: Phys. Chem. Chem. Phys., 2018, 20, 5827

Received 19th September 2017, Accepted 30th January 2018

DOI: $10.1039 / c 7 c p 06416 k$

rsc.li/pccp

\section{Origin of enhancement in Raman scattering from Ag-dressed carbon-nanotube antennas: experiment and modelling $\dagger$}

\author{
T. V. Raziman, (D) $\ddagger^{a}$ J. A. Duenas, $(\mathbb{D}) \S^{\mathrm{b}}$ W. I. Milne, ${ }^{\mathrm{C}}$ O. J. F. Martin and \\ P. Dawson (iD *b
}

\begin{abstract}
The D- and G-band Raman signals from random arrays of vertically aligned, multi-walled carbon nanotubes are significantly enhanced (up to $\sim 14 \times$ ) while the signal from the underlying Si substrate is simultaneously attenuated (up to $\sim 6 \times$ ) when the nanotubes are dressed, either capped or coated, with $\mathrm{Ag}$. These Ag-induced counter-changes originate with the difference in geometry of the nanotubes and planar Si substrate and contrast in the Ag depositions on the substrate (essentially thin film) and the nanotube (nano-particulate). The surface integral equation technique is used to perform detailed modelling of the electromagnetic response of the system in a computationally efficient manner. Within the modelling the overall antenna response of the Ag-dressed nanotubes is shown to underpin the main contribution to enhancement of the nanotube Raman signal with hot-spots between the $\mathrm{Ag}$ nanoparticles making a subsidiary contribution on account of their relatively weak penetration into the nanotube walls. Although additional hot-spot activity likely accounts for a shortfall in modelling relative to experiment it is nonetheless the case that the significant antenna-driven enhancement stands in marked contrast to the hot-spot dominated enhancement of the Raman spectra from molecules adsorbed on the same Ag-dressed structures. The Ag-dressing procedure for amplifying the nanotube Raman output not only allows for ready characterisation of individual nanotubes, but also evidences a small peak at $\sim 1150 \mathrm{~cm}^{-1}$ (not visible for the bare, undressed nanotube) which is suggested to be due to the presence of trans-polyacetylene in the structures.
\end{abstract}

\section{Introduction}

The field of surface enhanced Raman scattering (SERS) has seen the development of a huge variety of substrate architectures implemented by a range of fabrication methodologies, generally with the aim of optimising electromagnetic field enhancement at the surface and thus the Raman signal enhancement. The motivating rationale is an understanding that the electromagnetic enhancement is dominant and that chemical enhancement effects are subsidiary. ${ }^{1}$ We work within

\footnotetext{
${ }^{a}$ Nanophotonics and Metrology Laboratory, École Polytechnique Fédérale de Lausanne, CH-1015 Lausanne, Switzerland

${ }^{b}$ Centre for Nanostructured Media, School of Maths and Physics, Queen's University, Belfast BT7 1NN, UK. E-mail: p.dawson@qub.ac.uk ${ }^{c}$ Electrical Division Building, Engineering Department, Cambridge University, $9 \mathrm{JJ}$ Thomson Avenue, Cambridge CB3 OFA, UK

$\dagger$ Electronic supplementary information (ESI) available. See DOI: 10.1039/ c7cp06416k

$\$$ Both authors contributed equally.

$\S$ Present address: Departamento de Ingeniería Eléctrica, Escuela Técnica Superior de Ingeniería, Universidad de Huelva, 21819 Huelva, Spain.
}

this understanding in the present investigation. The goal in much substrate fabrication is the creation of surface structures that support localised plasmon resonances, particularly in nanogaps between $\mathrm{Au}$ or $\mathrm{Ag}$ particles, such as are present in the samples described here. The critical importance of the nanogap dimension in determining the magnitude of the Raman scattering from molecules located in such gaps has been demonstrated in experiment and modelling of single, optically isolated, plasmonic dipole antennas organised in an array where the antenna arm length and gap dimension were systematically varied in orthogonal directions. ${ }^{2}$ While there is a direct and clear correlation between the macroscopic, far-field response and the near-field Raman response at the level of the single antenna ${ }^{2}$ the relationship becomes much more subtle for practical SERS substrates where there is a distribution in nanogap dimension that generally occurs against a background of larger-scale surface structuring. ${ }^{3}$ Indeed, the targeting of highly localised plasmon resonances to drive large Raman enhancement often leads to the misconception that substrates displaying strong far-field plasmonic resonances (e.g. in reflection), should be effective SERS substrates. This is not the 
case - often a relatively small population of 'hot-spots' will dominate the Raman response ${ }^{4}$ but leave little imprint on the reflectance or transmittance. ${ }^{5}$ The relationship between the far-field optical and near-field SERS responses has been rigorously explored using $\mathrm{Au}$ nanowire arrays grown in an anodised alumina template as an exemplar system. ${ }^{6}$

In this investigation, we address the enhancement of the D- and G-band Raman signals from random arrays of vertically aligned, multi-walled carbon nanotubes (MWCNTs) dressed with a particulate Ag coating. The immediately relevant background is our use and analysis of such substrates to enhance Raman spectra from molecules deposited on the surface. ${ }^{7}$ But, before further consideration of the specific features of this substrate type we set the context by briefly surveying the structuring of some solid-state substrates used in SERS. For present purposes, we broadly classify such substrates into two categories, those with low-aspect ratio nanostructures and those having high aspect-ratio nano-structuring. The former include randomly rough surfaces, such as those formed by electrochemical etching in the seminal work of Fleischmann et $a l .{ }^{8}$ and various surfaces and nanoparticle (NP) arrangements displaying as-prepared fractal topology. ${ }^{9-11}$ Also included in this category is Klarite ${ }^{\mathrm{TM}}$, a commercial, dielectric-templated substrate comprised of periodic arrays of inverted microscale pyramids etched in $\mathrm{Si}$ and coated with a nanostructured $\mathrm{Au}$ film. This substrate has been used, for example, in quantitative SERS detection of explosive pre-cursors ${ }^{12-14}$ on account of good Raman reproducibility - a main selling point at a high price point, but a feature missing from very many of the SERS substrates reported in the research literature.

Substrates with high aspect-ratio structures include arrays of metal nanoneedles prepared by oblique angle deposition ${ }^{15}$ and metal nanorods grown electrochemically in anodised alumina templates. ${ }^{5,6,16,17}$ However, all-metal nanostructures tend to be mechanically and thermally fragile. Alternatively, $\mathrm{Au}$ and $\mathrm{Ag}$ dressings may be applied to high-aspect ratio structures such as arrays of nanocone $\mathrm{Si}$, either used directly ${ }^{18}$ (or for nanoimprinting into polymer substrates ${ }^{19}$ ), Si-nanopillars, ${ }^{20,21}$ glass nanopillars, ${ }^{22}$ polystyrene nanotubes ${ }^{23}$ and, of specific interest here, carbon nanotubes. ${ }^{7,24-29}$ The polymer-based structures ${ }^{19,23}$ tend to share the mechanical and thermal fragility of their all-metal counterparts ${ }^{5,6,15-17}$ in contrast to the metal-dressed $\mathrm{Si}^{20,21}$ glass $^{22}$ and carbon structures. ${ }^{7,24-29}$ A comprehensive review of plasmonic nanopillar arrays for application in SERS has recently been given by $\mathrm{Oh}$ et $a .^{3}{ }^{3}$ In brief, in addition to the requisite (near-field) optical properties, other key aspects of SERS substrates are good uniformity and reproducibility, as well as mechanical and thermal robustness.

In early work that utilized metal particle dressing of CNTs the focus was on analysis of the Raman spectra of the nanotubes themselves, ${ }^{30-36}$ while the weight of interest in later work has tended to be on their use as general SERS substrates ${ }^{7,24-29}$ with some investigations covering both aspects. ${ }^{29}$ However, while metal NP-dressed CNTs offer a mechanically and thermally robust framework for general SERS application, reproducibility is an issue due to the lack of control over the parameters of the
NP dressing, particularly the distribution in nanogap dimensions. (This generic problem of SERS substrates has been addressed by using very thin sacrificial dielectric spacers between metallic nanostructures, ${ }^{37-40}$ though even here there appear to be limitations on nanogap uniformity imposed by the metal grain structure. It is for this reason that we have recently proposed the use of hybrid waveguide-plasmon structures for SERS where the main hybrid-mode hotspot region is defined within a thin dielectric layer that is not sacrificially removed. ${ }^{41}$ ) Nonetheless, from the perspective of enhancing the Raman signal from MWCNTs themselves, the use of a random dressing of $\mathrm{Ag}$ or $\mathrm{Au}$ NPs is of great utility and experimentally straightforward to achieve. The modelling of this dressing and the analysis of the electromagnetic response of the MWCNT structures has been a major motivating challenge of this work.

To set the range of Raman enhancement factors (EF), Ag-dressed MWCNTs yield an EF of $10^{3}-10^{4}$ for molecules deposited on the surface (estimated EF $\sim 3 \times 10^{4}$ for the first monolayer ${ }^{7}$ while the underlying Raman signature of the MWCNTs themselves has an EF $\sim 10$ (detail to follow); in our related work with molecular deposits on arrays of $\mathrm{Au}$ nanorods and nanotubes ${ }^{5,6}$ the EF is $\sim 10^{3}$ where the same calibration procedure has been used throughout. The wide range of EF reflects differences in the underlying near-field optical responses of the systems and the location of the Raman active entities with respect to regions of high near-field concentration. For the smaller scale, closely packed Au-nanorod/nanotube systems, transverse, longitudinal and cavity mode localised plasmon resonances are the key drivers. ${ }^{6}$ These substrates, like the (metal NP-dressed) MWCNT substrates display strong coloration effects in the far-field (reflectance), characteristic of an optical antenna array. However, for Ag-dressed MWCNTs the Raman enhancement of deposited molecules derives from multiple hotspots (i) on Ag NPs (amplified by the antenna response), (ii) between $\mathrm{Ag}$ particles and (iii) between the tops of co-leaning nanotubes. ${ }^{7}$

When it comes to the Raman enhancement of the D- and G-bands of the MWCNTs underlying the Ag dressing, it should not be assumed, a priori, either that the coloration is an indicator of good Raman enhancement (coloration is a farfield effect, after all) or that hotspots are the main driver of that enhancement. The Raman signature of the MWCNTs is always present - although enhanced with respect to that of bare MWCNTs it is typically 2 to 3 orders of magnitude less intense than those of molecular adsorbates on the Ag-dressing. This enhanced 'background' Raman scattering is of significant interest on a number of grounds: (i) its origins, particularly the electromagnetic component which is analysed in rigorous detail in this study; (ii) as a means to more sensitively probe and characterise carbon nanotubes; (iii) as means of rendering the probing of individual nanotubes within the array feasible (or more readily accessible) and (iv) as an exemplar system for enhanced Raman characterisation of other, similarly dressed vertical nanorod/nanotube entities with crystalline structure.

As in our previous work ${ }^{7}$ the electromagnetic response is analysed using the surface integral equation (SIE) technique ${ }^{42}$ - further 
detail is outlined in Methods. Understanding the performance of many effective Raman enhancing substrates is often hampered by the fact that they are structurally very complex and so not amenable to quantitative modelling and analysis. In such situations, the analysis can focus on certain features e.g. a specific type of hotspot region, which are assumed to drive the Raman signal. Here such an assumption could be quite inappropriate and it is a key feature of this investigation that the computational efficiency of the SIE technique facilitates treatment of the Ag-related hot-spots within a 3D analysis of the overall MWCNT antenna structures. The method is significantly more computationally efficient than, for example, application of commercial finite-element or finite-difference timedomain software packages.

\section{Experiment and results}

The background SEM and (elastic) optical data of the MWCNT samples are summarised in Fig. 1, with the core Raman spectroscopy results given in Table 1 and Fig. 2. Detailed modelling and analysis of the Raman response is presented in the next section. The substrates comprise of random arrays of vertically aligned MWCNTs which are typically fabricated to be $\sim 400 \mathrm{~nm}$ to several micron in length and $>50 \mathrm{~nm}$ diameter. The MWCNTs of the substrates examined here, as shown in the inset to Fig. 1a, have an average length, $L_{\mathrm{NT}}=495 \pm 16 \mathrm{~nm}$ and radius, $R_{\mathrm{NT}}=32.2 \pm 2.2 \mathrm{~nm}$. (Note, in arriving at these average
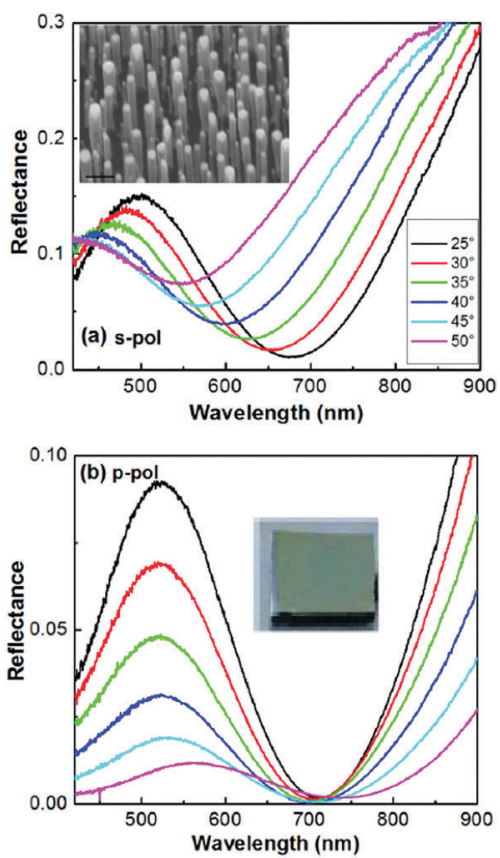

Fig. 1 (a) Specular reflectance of s-polarised light from vertically aligned MWCNT sample for angles of incidence shown in the key. Inset - SEM image of the sample taken at $\sim 45^{\circ}$; Ni catalyst particles are clearly visible at the tops of the nanotubes. Average nanotube length and radius are $L_{\mathrm{NT}}=495 \pm 16 \mathrm{~nm}$ and $R_{\mathrm{NT}}=32.2 \pm 2.2 \mathrm{~nm}$. (b) Specular reflectance of p-polarised light with same colour key as (a); inset - photograph of the sample showing a green-blue coloration consistent with reflectance spectra. figures which feed through to the modelling we have ignored the significantly shorter and thinner nanotubes, some of which can be seen in this image.) The MWCNT forests were fabricated using plasma-enhanced chemical vapour deposition (PECVD) in the manner originally described by Chhowalla et $a l^{43}$ and developed by Teo et al.; ${ }^{44}$ further details are given under Methods. The hollow nature of MWCNTs has been clearly illustrated and characterised in TEM analysis by various groups. ${ }^{43,45-47}$ The overall MWCNT diameter and wall thickness can vary somewhat depending on the preparation conditions, with smaller diameter MWCNTs (less than $\sim 20 \mathrm{~nm}$ ), prepared using (non-plasma-assisted) CVD methods, showing a wall thickness, $W_{\mathrm{NT}}$, of the order of $5 \mathrm{~nm}^{46,47}$ while the MWCNTs reported by Sun et al. ${ }^{45}$ have $R_{\mathrm{NT}} \sim 30 \mathrm{~nm}$, similar to those shown in Fig. 1a, and $W_{\mathrm{NT}} \sim 10-12 \mathrm{~nm}$. For the purpose of the modelling presented below we have chosen $W_{\mathrm{NT}}=10 \mathrm{~nm}$, yielding a fill factor just under 50\%. Also evident in the SEM micrograph of Fig. 1a are the Ni catalyst particles enclosed in a carbon sheath at the top of the nanotubes.

These vertically-aligned MWCNT samples display strong, length-dependent coloration effects with that shown in the inset of Fig. 1a exhibiting a green-blue colour in reflection (Fig. 1b, inset). The specular reflectance in s-polarisation has a broad peak centred close to $\lambda=500 \mathrm{~nm}$ for light incident at $25^{\circ}$ and moves to shorter wavelengths with increasing angle of incidence (Fig. 1a). In contrast, the peak in p-polarised reflectance remains almost constant in wavelength as a function of incident angle. This is consistent with the optical properties of such systems described in our previous work ${ }^{7}$ where the s-polarised reflectance was analysed in terms of interference between light reflected at the surface of the nanotube 'layer' (treated in an effective medium model) and that reflected from the substrate. The nanotube layer is highly anisotropic with the p-polarised reflectance being dictated by the antenna response along the length of the MWCNTs.

We turn now to the main focus of our attention, the Raman response as detailed in Fig. 2 for native MWCNTs and for MWCNTs with two different types of $\mathrm{Ag}$ dressing. The first is produced by a normal-incidence thermal evaporation of $7 \mathrm{~nm}$ of $\mathrm{Ag}$ which forms a layer of $\mathrm{Ag}$ on the Si substrate but just 'caps' the MWCNTs, with no obvious deposition on the sidewalls (Fig. 2a inset). The second is performed by Ag evaporation at an angle of $\sim 15^{\circ}$ to the substrate normal with the substrate being spun about its axis. This results in a Ag deposit on top of the nanotubes (thickness is $18 \mathrm{~nm}$ in the case of sample of Fig. 2d-f), with a significant amount of highly granular Ag attached to the MWCNT sidewalls, as well as a coating on the $\mathrm{Si}$ substrate (Fig. 2d, inset). In both cases there is a remarkable contrast between pre- and post-Ag deposition in that the Raman signal from the $\mathrm{Si}$ substrate is attenuated with $\mathrm{Ag}$ dressing (Fig. 2a and d) while that of the MWCNT D- and G-bands is significantly enhanced (Fig. 2b and e). Fig. 2c and $\mathrm{f}$ map the intensity of the Si peak/MWCNT D-, G-bands as a function of position on the surface where the input laser beam was scanned across a diameter of the Ag-treated spot. In the case of the Ag-capped sample the transition from the Ag-treated 
Table 1 Enhancement factors, EF, for MWCNT Raman signals and attenuation factors, AF [in square brackets], for Si-substrate Raman signals for model systems as denoted in left-most column and described in Fig. 3 and the text. In the top row (Bare MWCNTs) EF is set to unity with absolute value of $\left|\mathbf{E}_{\text {loc }}\right|^{4}$ given assuming input field of unit intensity $\left(1 \mathrm{~V} \mathrm{~m}^{-1}\right)$. The corresponding experimental data are also given where EF and AF are evaluated from the areas beneath the spectra shown in Fig. 2 in the range $507-531 \mathrm{~cm}^{-1}$ (Si-peak) and $1200-1700 \mathrm{~cm}^{-1}$ (MWCNT D- and G-bands, i.e. excluding the region with the subsidiary peak at $\sim 1150 \mathrm{~cm}^{-1}$ ). There is no Ni sphere at the top of the nanotube included in the modelling for the data in this table

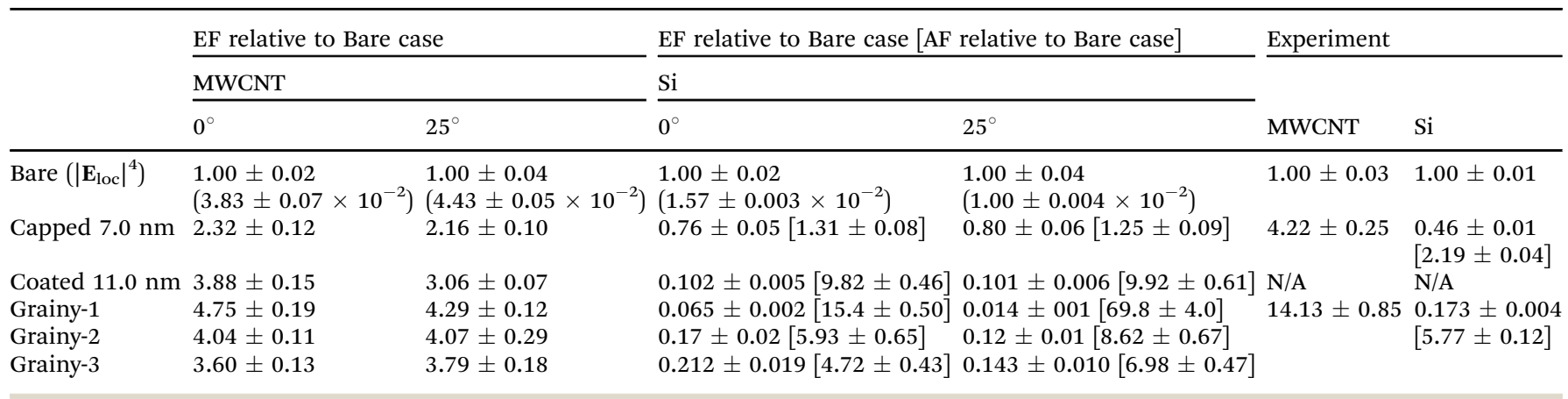

to native MWCNT regions is abrupt (Fig. 2c) while for the offnormal evaporation there is a penumbra region in the $\mathrm{Ag}$ deposition such that the Raman signal from the Si decreases while that from the MWCNTs increases progressively in going from the uncoated to the fully Ag-coated region (Fig. 2f). Relative to the adjacent, 'bare' MWCNT region of the sample the Ag-capped region yields a Si-peak attenuation of $\sim 2.2$ and D-, G-band enhancement of $\sim 4.2$, while the corresponding values for the sample with the fully-coated $\mathrm{Ag}$ region are an attenuation of 5.8 and enhancement of 14.1, yielding discrimination of $\sim 9.2 \times$ and $\sim 82 \times$ respectively in favour of the MWCNT Raman signature over that of the Si substrate. These data are recorded in Table 1 along with the corresponding modelled data.

The measured length, $L_{\mathrm{NT}(\mathrm{Ag})}$, and radius, $R_{\mathrm{NT}(\mathrm{Ag})}$, of the $\mathrm{Ag}$ capped MWCNTs are $511 \pm 15$ and $33.2 \pm 2.5 \mathrm{~nm}$ respectively, while for the Ag-coated samples $L_{\mathrm{NT}(\mathrm{Ag})}=518 \pm 16 \mathrm{~nm}$ and $R_{\mathrm{NT}(\mathrm{Ag})}=43.0 \pm 2.7 \mathrm{~nm}$. Not surprisingly, there is some discrepancy between $L_{\mathrm{NT}}$ with the nominal $\mathrm{Ag}$ thicknesses added and the values actually recorded for the Ag-dressed samples. This is in part due to the fact that the various samples or chips come from different regions of the Si wafer. Giving stronger weighting to the data for the Ag-dressed samples we decided, for the purposes of the modelling, to round $L_{\mathrm{NT}}$ up from $495 \mathrm{~nm}$ to $500 \mathrm{~nm}$, but retain $R_{\mathrm{NT}}=32 \mathrm{~nm}$.

As a point of reference, in concluding this section it is noted that the EFs for the Ag-dressed MWCNTs found here are in line with most of those recorded in other Raman investigations involving the attachment of $\mathrm{Au}$ and $\mathrm{Ag}$ NPs to CNTs. ${ }^{30-36}$ Further comment on the comparative performance these substrates will be given later.

\section{Modelling and analysis}

Fig. 3 illustrates the meshes used in the simulations, starting with the bare MWCNT in Fig. 3a. For the Ag-capped structure (Fig. 3b), depicting the case of MWCNTs subject to normal incidence $\mathrm{Ag}$ evaporation, a cap of $7.0 \mathrm{~nm} \mathrm{Ag}$ is added to the hemispherical top of the MWCNT and a film of the same thickness to the Si substrate; the radius is taken to be $32 \mathrm{~nm}$, the same as for the bare MWCNT sample i.e. discounting the measured value of $33.2 \mathrm{~nm}$ which lies within the margin of error in the measurement. The off-normal evaporation of $\mathrm{Ag}$ onto a spinning substrate yields a highly granular, nanoparticulate coverage of the MWCNTs (inset SEM image of Fig. 2d) where $R_{\mathrm{NT}(\mathrm{Ag})}=43 \mathrm{~nm}$, i.e. to the outside edges of the NPs is $11 \mathrm{~nm}$ greater than for the case of the bare MWCNT. En route to developing a model of such grainy Ag structures we consider a structure comprised of MWCNTs uniformly coated with $11 \mathrm{~nm}$ $\mathrm{Ag}$, the Coated structure of Fig. 3c. This Ag coating is, of course, thicker than would result from conformal thin film coating of the MWCNT but this case serves as a useful reference for the Grainy structures of Fig. $3 \mathrm{~d}-\mathrm{f}$ where the Ag grains are modelled as spheres of radius, $r$, distributed with their centres uniformly distributed along a helix with the central axis at a distance, $R$, from the MWCNT axis; only those sections of the spheres beyond the boundary of the MWCNT are Ag. This yields a pattern of Ag 'protrusions' of spherical section, such that 12-13 such features are encountered in a vertical trajectory along the length of the nanotube walls, mimicking the structures of Fig. 2d. A range of grainy structures was modelled with the following parameters: $r=27 \mathrm{~nm}, R=16 \mathrm{~nm}$ (Grainy-1, Fig. 3d); $r=22.3 \mathrm{~nm}, R=20.7 \mathrm{~nm}$ (Grainy-2, Fig. $3 \mathrm{e}$ ); $r=19.5 \mathrm{~nm}$, $R=23.5 \mathrm{~nm}$ (Grainy-3, Fig. 3f). In all cases, the maximum extent of the $\mathrm{Ag}$ grains beyond the nanotube wall is $11 \mathrm{~nm}$. Thus, in going from the smoothly coated structure of Fig. 3c to Grainy-1, -2 and -3 (Fig. $3 \mathrm{~d}-\mathrm{f}$ ) the amount of $\mathrm{Ag}$ is progressively reduced while the overall radius to the outer edges of the structures remains constant at $43 \mathrm{~nm}$. The $\mathrm{Ag}$ coverage on Grainy- 1 is markedly profiled but remains virtually continuous (there are a few pinholes, not visible in Fig. 3d) while Grainy-3 exhibits smaller, clearly separated protrusions; in Grainy-2 the Ag protrusions just overlap, but few nm gaps also exist between adjacent grains, exposing small areas of the underlying MWCNT. In a further, realistic physical refinement we have also made provision for the inclusion of a Ni sphere encapsulated in the hemispherical top. Finally, note that the modelling 

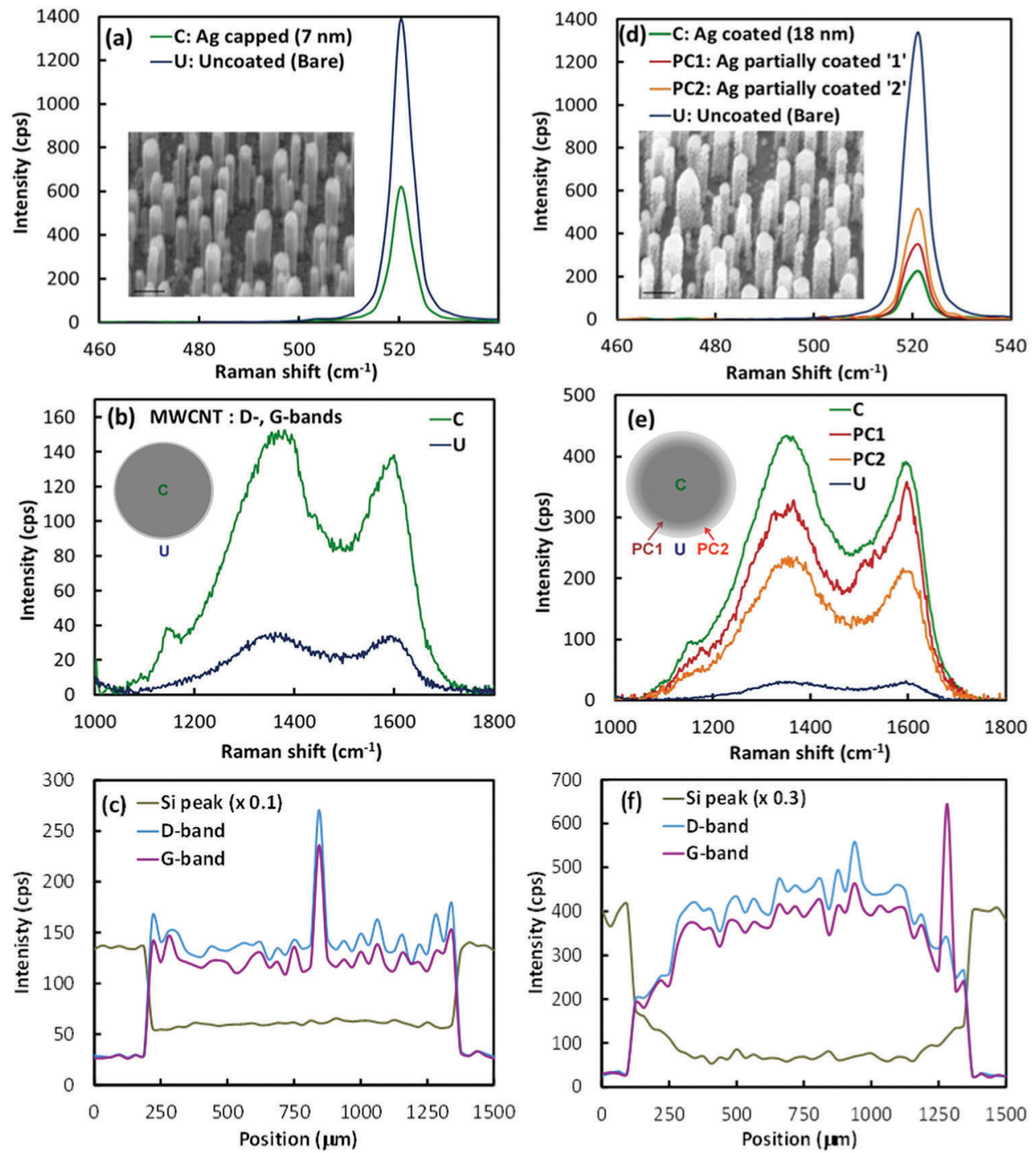

Fig. 2 (a) Raman spectrum in the range $460-540 \mathrm{~cm}^{-1}$, showing Si peak at $521 \mathrm{~cm}^{-1}$ for bare MWCNT-Si substrate and for different area of same substrate coated with $7.0 \mathrm{~nm} \mathrm{Ag}$ at normal incidence; inset shows SEM image of coated substrate taken at $\sim 45^{\circ}$ (scale bar is $200 \mathrm{~nm}$ ). (b) Section of same spectra as for part (a), in range 1000-1800 $\mathrm{cm}^{-1}$, showing D- and G-bands of MWCNTs. (c) Intensity of Si-peak and D- and G-bands for Ag-capped sample of part (a) as function of position spanning diameter of Ag-dressed MWCNTs. (d-f) Equivalent data for case of sample with $18 \mathrm{~nm}$ Ag deposited over area of $\sim 1.2 \mathrm{~mm}$ diameter at angle of $\sim 15^{\circ}$ with respect to the substrate normal on to rotating sample with different regions of sample as specified in the inset to (e). Raman spectra were taken with Jobin-Yvon LabRam microscope with $50 \times$ objective; $\lambda=632.8 \mathrm{~nm}, P_{\text {in }}=2 \mathrm{~mW}$; acquisition performed in $2 \times 3$ s captures. Raman spectrum was taken at each of 200 points in the spatial scans of (c) and (f).

assumes a regular square array of nanotubes of period $160 \mathrm{~nm}$ in $x$ and $y$ to accommodate the effect of coupling between the nanotubes at the measured areal density.

The modelled optical response of the nanotubes is presented in Fig. 4-7. The bulk of the analysis is conducted with reference to Fig. 4-6 in which the (hollow) nanotubes do not have the Ni inclusion. There are two reasons for initially overlooking the Ni inclusions. First, at the cost of minor simplification, all the key features of the optical response are captured in the analysis of the hollow, Ag-dressed MWCNTs without the Ni inclusion. The second reason is that the introduction of the $\mathrm{Ni}$ spheres leads to a region of extreme geometrical constriction and field confinement between the sphere and the inner wall of the MWCNT that produces large fluctuations in the modelled data. The influence of the $\mathrm{Ni}$ inclusions is subsequently addressed with reference to Fig. 7 .

Fig. 4a shows cross-sections in the $x-z$ diametric plane of the local field intensity variation, $\left|\mathbf{E}_{\mathrm{loc}}\right|^{2}$, assuming an incident field of unit magnitude, for the various MWCNT structures. The input light is of wavelength $633 \mathrm{~nm}$ incident in the $-z$-direction (i.e. angle of incidence $=0^{\circ}$ ) with the polarisation vector in the $x$-direction where the axes are as specified in Fig. 3. Fig. $4 \mathrm{~b}$ illustrates the same data on a log-intensity scale which effectively 'stretches' the intensity scale to better differentiate between and within regions of low and high intensity. Fig. 5a shows the equivalent log-scale intensity plots for $633 \mathrm{~nm}$, p-polarised light incident on the same structures at an angle of $25^{\circ}$ (i.e. coming from the top-left of the figure). This offers a 
(a) Bare

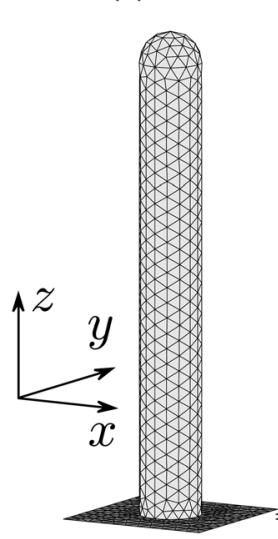

(b) Capped

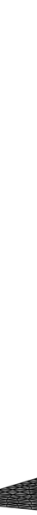

(c)
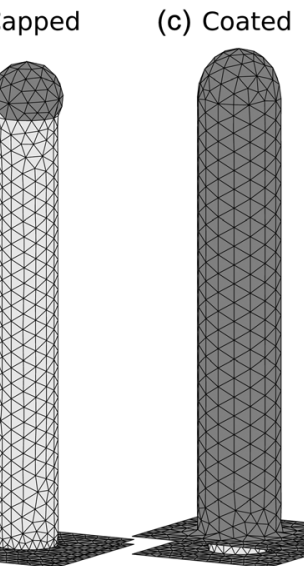

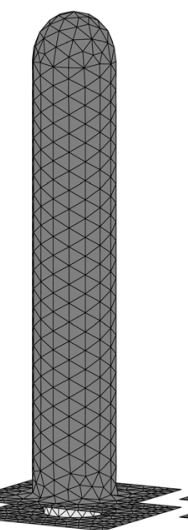

(d)

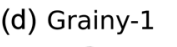

(e)
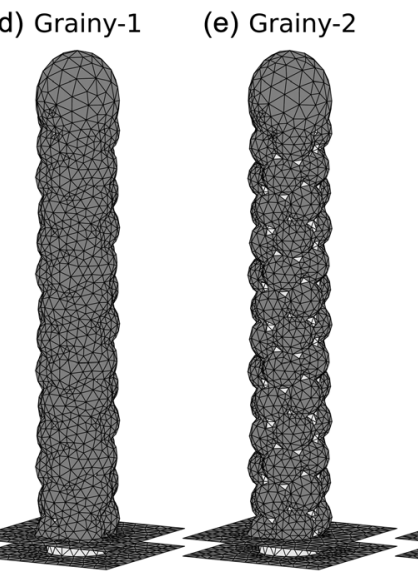

(f) Grainy-3

Fig. 3 Simulation geometries of MWCNTs with different Ag dressings - in all cases $L_{N T}=500 \mathrm{~nm}, R_{\mathrm{NT}}=32 \mathrm{~nm}$ and $W_{\mathrm{NT}}=10 \mathrm{~nm}$ and the Si substrate unit cell is $160 \times 160 \mathrm{~nm}$ (a) Bare: MWCNT with no Ag dressing; (b) Capped: MWCNT with $7 \mathrm{~nm}$ Ag coating on hemispherical top and on Si substrate; (c) Coated: MWCNT with $18 \mathrm{~nm}$ Ag coating on top and on Si substrate and $11 \mathrm{~nm}$ coating on the sidewalls $\left(R_{\mathrm{NT}(\mathrm{Ag})}=43 \mathrm{~nm}\right)$; (d) Grainy-1, (e) Grainy-2 and (f) Grainy-3 depict MWCNTs with $18 \mathrm{~nm} \mathrm{Ag} \mathrm{coating} \mathrm{on} \mathrm{top} \mathrm{and} \mathrm{on} \mathrm{Si} \mathrm{substrate,} \mathrm{with} \mathrm{sidewalls} \mathrm{dressed} \mathrm{with} \mathrm{Ag} \mathrm{particles} \mathrm{of} \mathrm{spherical} \mathrm{profile} \mathrm{such} \mathrm{that} R_{\mathrm{NT}(\mathrm{Ag})}$ remains constant at $43 \mathrm{~nm}$; details of the geometrical construction are given in the text.

spot check for light that is non-normally incident on the sample through the microscope objective (numerical aperture 0.55 , yielding a cone of incident light of half angle $34^{\circ}$ ) where the important physical distinction is that there is now a component of electric field parallel to the MWCNT axis.

The key parameter in analysing the Ag-induced enhancement of the Raman signal from the MWCNTs (and the attenuation of that from the $\mathrm{Si}$ substrate) is $\left|\mathbf{E}_{\mathrm{loc}}\right|^{4}$ within each material. ${ }^{48}$ Here, we make the common approximation of evaluating $\left|\mathbf{E}_{\text {loc }}(\omega)\right|^{4}$ at the incident frequency, $\omega$, rather than the product of $\left|\mathbf{E}_{\text {loc }}(\omega)\right|^{2}$ and $\left|\mathbf{E}_{\text {loc }}(\omega-\Delta \omega)\right|^{2}$ where $\Delta \omega$ is the Stokes shift and is a function of the excitation mode. (This saves the considerable computational effort of evaluation at small $\omega$-intervals over the large spread in $\Delta \omega$ of the experimental data.) The plots of $\left|\mathbf{E}_{\text {loc }}(\omega)\right|^{4}$ of Fig. $4 \mathrm{c}, \mathrm{d}$ and $5 \mathrm{~b}$ are based on a sample of 40000 points within the volume of interest using a Monte Carlo approach, as detailed in the Methods section. For ease of visualisation in the case of the nanotube walls (Fig. $4 \mathrm{c}$ and $5 \mathrm{~b}$ ) all the points have been rotated on to the negative $x-z$ half-plane. Also shown in Fig. $4 \mathrm{~d}$ is a plot of $\left|\mathbf{E}_{\text {loc }}\right|^{4}$ for the Si substrate where the modelled volume $\left(160 \times 160 \times 100 \mathrm{~nm}^{3}\right.$ in $x, y$ and $z$ respectively $)$ has been truncated at the $y-z$ plane, compressed to the $x-z$ plane and juxtaposed with respect to the images of $\left|\mathbf{E}_{\text {loc }}\right|^{4}$ for the MWCNTs of part (c). The $\left|\mathbf{E}_{\text {loc }}\right|^{4}$ plots in the Si substrate for the case of $25^{\circ}$ incidence (not shown) are virtually identical to those of Fig. $4 \mathrm{~d}$.

We now consider the salient features in both the elastic and Raman regimes with reference to Fig. 4 and 5. The overarching feature in all the intensity cross-sections is a simple standing wave pattern along the length of the antenna with two pronounced anti-nodes bounded by nodal regions at the bottom, top and in the middle of the antenna. Consider first the Bare and Coated cases. While the Bare MWCNT has an average metallic character in terms of dc conductivity its optical character is that of a lossy dielectric where we take the refractive index, $n_{\mathrm{NT}}=3.0+i(C / 3.0) \lambda$ with $C=5.445 \mu \mathrm{m}^{-1}$, i.e. $3.0+i 1.15$ here, after Bruna and Borini, ${ }^{49}$ as used in our previous analysis of MWCNT antenna structures. ${ }^{7}$ These structures act to confine the applied EM fields (both incident and reflected) along their length in a standing wave pattern (Fig. $4 a, b$ and 5a) that would otherwise be invariant in the $x-y$ plane in the case of a planar reflecting surface with no antenna structures. The Coated structure is a plasmonic antenna in the sense that it possesses a shell of free-electron gas material but is not plasmonically resonant at $633 \mathrm{~nm}^{50}$ Nonetheless, relative to the Bare MWCNT, the introduction of the smooth $11 \mathrm{~nm}$ thick Ag-coating yields an enhanced field intensity pattern that is of slightly shorter period. The other structures, i.e. the Ag-Capped and Ag-Grainy structures, introduce local, nano-scale variations within the overall standing wave pattern, discussed further below. Paradoxically, however, the standing waves associated with the Ag-Grainy MWCNT antennas have a wavelength that is closer to that of the Bare structure than the Coated MWCNT.

For the Bare structure in Fig. 4c, the Raman intensity is modulated along the length of the tube following the overall antenna intensity pattern. The Bare case forms the baseline against which the Raman enhancement of the other structures is referenced, but the term is used advisedly since $\left|\mathbf{E}_{\text {loc }}\right|^{4}$ is less than unity throughout much of the volume of the MWCNTs. The Capped structure exhibits the same general standing wave profile but with significant enhancement near the top where Ag has been deposited. The Coated structure shows stronger enhancement throughout the length of the tube, with the vertical spatial variation again following that of the standing wave pattern. However, in contrast to the Bare and Capped cases, the greatest enhancement occurs along the sidewalls of the MWCNT at the expense of Raman signal generated from the top of the MWCNT, which now lies in a more distinctly nodal region optically. Interestingly, also, points showing higher Raman intensity are situated towards the inside of the MWCNT. This suggests that the nanotube is not passively receiving the 
(a)

(b)
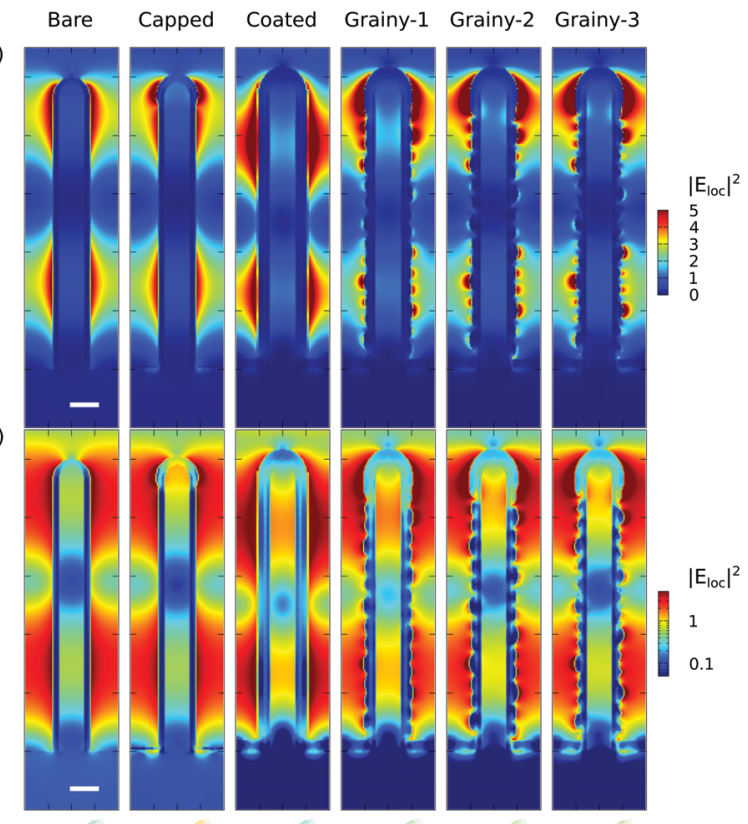

(c)
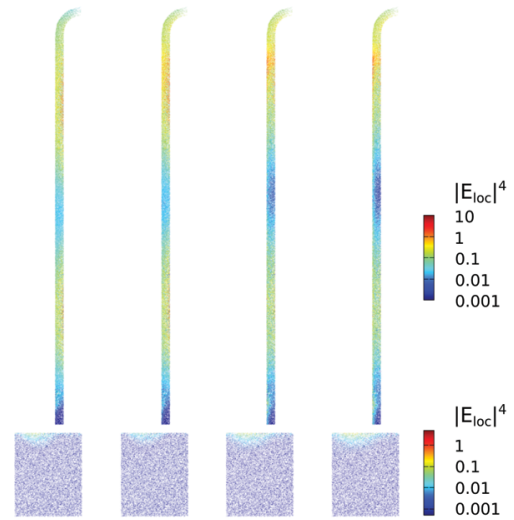

Fig. 4 Cross-sectional plots of the modelled (elastic) optical and Raman responses due to light of wavelength $632.8 \mathrm{~nm}$, polarised parallel to the $x$-direction, normally incident on bare and Ag-dressed MWCNTs as labelled above and described in Fig. 3; scale bars are $50 \mathrm{~nm}$ in all cases. (a) Cross-sectional plots in the $x-z$ plane of local intensity, $\left|E_{l o c}\right|^{2}$. The colour scale is saturated - maximum intensity is $>10$ - in order to offer good contrast in regions of interest. (b) Cross-sectional plots of part (a) shown on a log intensity scale. (c) Values of $\left|E_{\text {loc }}\right|^{4}$ at 40000 points selected inside the nanotube in the Monte Carlo approach; all points have been rotated around the central symmetry axis of the MWCNTs to lie in the negative $x-z$ half-plane. (d) Values of $\left|E_{\text {loc }}\right|^{4}$ based on 40000 points selected inside a volume of area $160 \times 160 \mathrm{~nm}$ and $100 \mathrm{~nm}$ depth ( $z$-direction) inside the Si substrate in the Monte Carlo approach; points shown lie in the half-volume for which $x \leq 0$ and have been collapsed to the $y-z$ plane. Images are juxtaposed with respect to those of $\left|\mathbf{E}_{\text {loc }}\right|^{4}$ for the MWCNTs of part (c), such that the right hand edge lies in line with the central axis of the MWCNTs.

intensity enhancement from the Ag-coating but acts to some extent as an optical cavity, thus playing a role in determining the field distribution. It therefore matters that the tube is hollow and not filled.

Consider next the Grainy Ag samples. The introduction of nanoscale structuring to the Ag dressing on the sidewalls of the nanotube, manifest as well-separated Ag NPs in the case of the Grainy-3 samples, leads to obvious hot-spots in $\left|\mathbf{E}_{\text {loc }}\right|^{2}$, notably

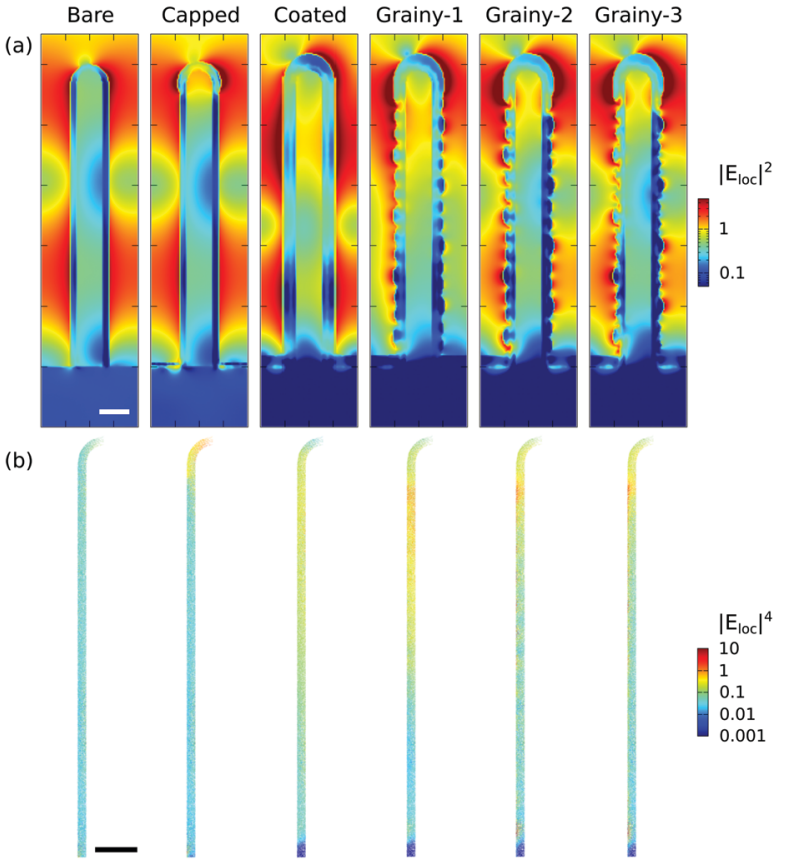

Fig. 5 Cross-sectional plots describing the (elastic) optical and Raman responses due to p-polarised light of wavelength $632.8 \mathrm{~nm}$, incident at an angle of $25^{\circ}$ with respect to the surface normal (i.e. from top left) on bare and Ag-dressed MWCNTs as labelled above and described in Fig. 3; scale bars are $50 \mathrm{~nm}$. (a) Cross-sections in the $x-z$ plane of local intensity, $\left|\mathbf{E}_{\text {loc }}\right|^{2}$ plotted on log scale. (b) Values of $\left|E_{\mid o d}\right|^{4}$ at 40000 points selected inside the nanotube in the Monte Carlo approach; all points have been rotated around the central symmetry axis of the MWCNTs to lie in the negative $x-z$ half-plane.

at the outer extremities of the Ag grains, but also between the grains. These more intense regions occur within the modulating envelope of the antenna response. What is also striking in the images of Fig. $4 \mathrm{c}$ and $5 \mathrm{~b}$ is that the antenna maxima in $\left|\mathbf{E}_{\text {loc }}\right|^{4}$ migrate from the inner to the outer wall of the MWCNTs in going from Grainy-1 to Grainy-3 and, for the case of p-polarised light incident at $25^{\circ}$ only, that there is also an accompanying development of 'warm spots' on the outer side of the MWCNT. The detail is given in Fig. 6 which shows, on an enlarged scale, $\left|\mathbf{E}_{\text {loc }}\right|^{2}$ and $\left|\mathbf{E}_{\text {loc }}\right|^{4}$ for Grainy-2 for the case of $0^{\circ}$ and $25^{\circ}$ (p-pol) incidence. Fig. 6a shows a smoothly varying antenna-type pattern of $\left|\mathbf{E}_{\text {loc }}\right|^{4}$ within the MWCNT with the only notable hot region being near the 'neck' of the structure, while Fig. 6b illustrates correlation of the warm spots in $\left|\mathbf{E}_{\text {loc }}\right|^{4}$ with the inter-particle gap regions of the grainy $\mathrm{Ag}$ structure. These warm spots are clearest for Grainy-2 (Fig. 5b and 6b) with the main contribution clearly originating from inter-particle gaps on the incident side of the structure; in Grainy-1 they are not fully developed and in Grainy-3 they weaken as the gaps between Ag particles open up. However, from Table 1, which lists the average value of $\left|\mathbf{E}_{\text {loc }}\right|^{4}$ within the sampled MWCNT volume, it is evident that the warm spots are not the major driver of Raman enhancement of the D- and G-bands. While their development is seen to improve the Raman signal relative to the $11 \mathrm{~nm}$ Coated case it is notable that the $\left|\mathbf{E}_{\text {loc }}\right|^{4}$ response 
(a)

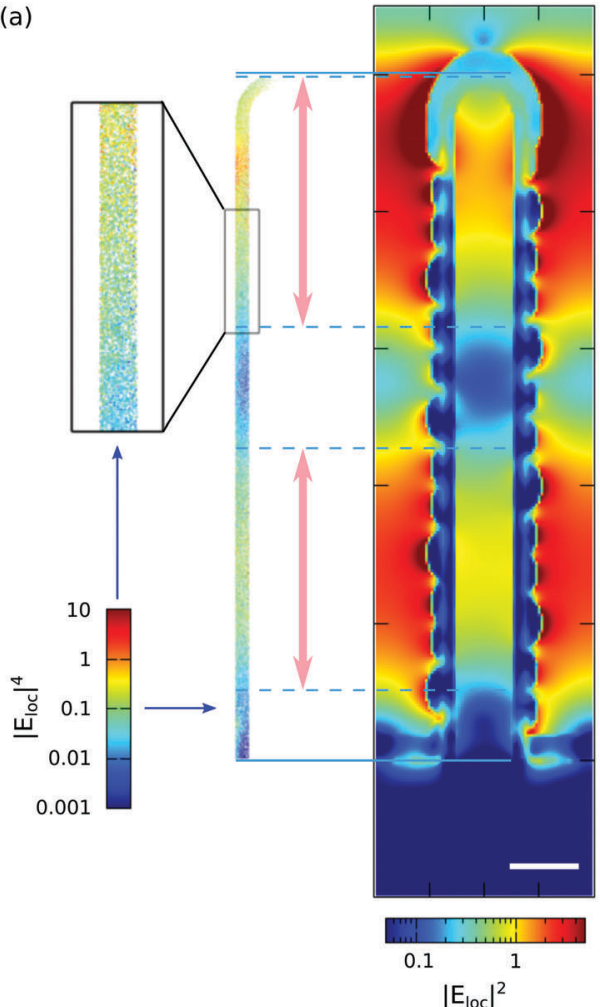

(b)

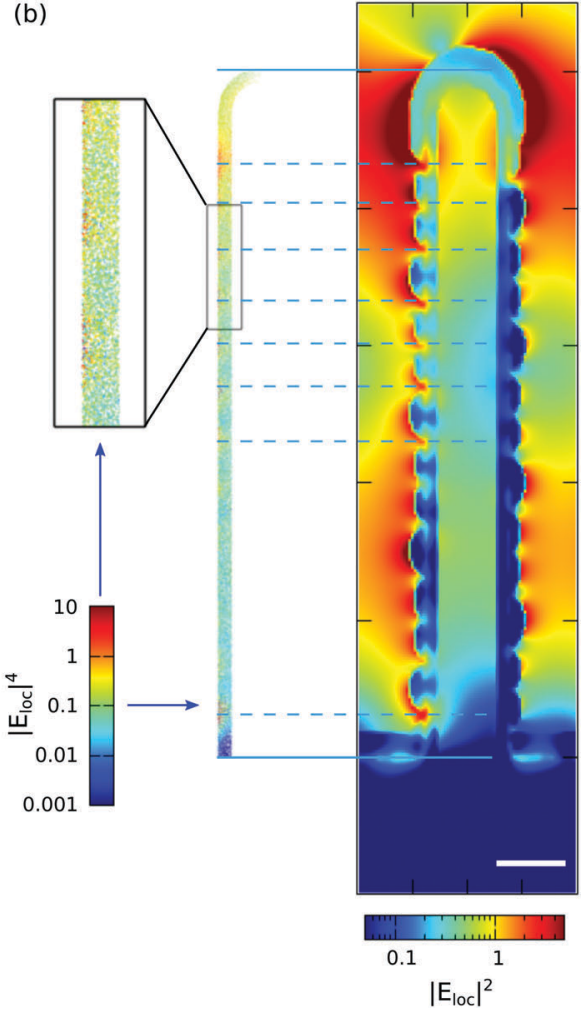

Fig. 6 (a) Main image shows cross-section of variation of $\left|\mathbf{E}_{\text {loc }}\right|^{2}$ for light of wavelength $633 \mathrm{~nm}$ incident at $0^{\circ}$, polarised in the $x$-direction (orthogonal to long axis of system), on sample Grainy-2. To the left is juxtaposed a plot of $\left|\mathbf{E}_{\text {loc }}\right|^{4}$, constructed in the manner of Fig. $4 \mathrm{c}$ and $5 \mathrm{~b}$, with a magnified view of a region towards the top of the MWCNT. The dashed lines, with pink double-headed arrow inserts, demarcate the main antenna anti-nodal regions. (b) Equivalent data for Grainy-2 sample with p-pol light incident at $25^{\circ}$ to the nanorod long axis (from top left) where dashed horizontal lines are drawn through gaps between the Ag grains.

of Grainy-1 is actually slightly superior to that of Grainy-2 and -3 . But, the performance of Grainy-1 is actually underpinned by a better penetration of the overall antenna fields into the MWCNT. This effect can also be achieved by a sample with a thinner $(7.5 \mathrm{~nm})$ conformal $\mathrm{Ag}$ coating which has also been modelled to reveal average $\left|\mathbf{E}_{\text {loc }}\right|^{4}$ values $\sim 5 \%$ higher than those for Grainy-1 and $\sim 16 \%$ higher than those for Grainy- 2 . When considered against the magnitude of the Raman signal generated by inter-Ag particle hotspots from molecules deposited on very similar, Ag-dressed MWCNTs $^{7}$ the warm spots uncovered here are just that - warm. In effect, the enhanced fields associated with hotspots between Ag NPs are extremely well confined to the inter-Ag region itself with only modest 'leakage' into the body of the MWCNT.

Experimentally there is significant reduction of the Raman signal from the Si substrate with Ag deposition (Fig. 2) and this is also observed in the modelling. The signal is strongest for the case of the Bare sample with a reduction of $20-25 \%$ in the modelled intensity for the Capped samples (see Table 1), a reduction which is not qualitatively evident from the images of Fig. 4d. However, what is clear from Fig. 4d is the general reduction in the Raman signal from the Si substrate for the Coated and Grainy samples where it is seen that only the region beneath the $\mathrm{Ag}$ sidewalls of the MWCNT retains the intensity of the Bare sample.
In summary, the enhancement of the Raman signal from the MWCNTs in parallel with the attenuation of that from the $\mathrm{Si}$ substrate with Ag deposition derives primarily from the different geometries of the two materials and to a subsidiary degree with the contrasting character of the Ag deposits on MWCNT and Si. The Ag coating of the MWCNTs, whether smooth or granular, has the effect of 'harvesting' the incident light, concentrating the standing wave fields along the nanotube structure (particularly near the top end of the structure), in effect enhancing the efficacy of the MWCNT as an optical antenna. Penetration of the EM fields into the MWCNT walls, along with the reciprocal re-emission process facilitates the retrieval of a Raman signal from the MWCNT. On the other hand the planar nature of the Si substrate, on which the Ag deposits essentially as a thin film, means that penetration of incident light through the film and of Raman scattered light back out is significantly attenuated. Returning to the MWCNTs, modelling indicates that an optimal thickness of conformal Ag coating will give a significant Raman EF but, given that the coating is particulate in practice, it is evident that there is a contribution to the Raman signal from inter-particle warm spots on the outer side of the nanotube walls. Ag capping of the MWCNTs tends to focus energy in the cap-region, also enhancing the Raman output.

We now consider the values of the MWCNT EF and Si substrate attenuation factor, AF. A preliminary observation is 

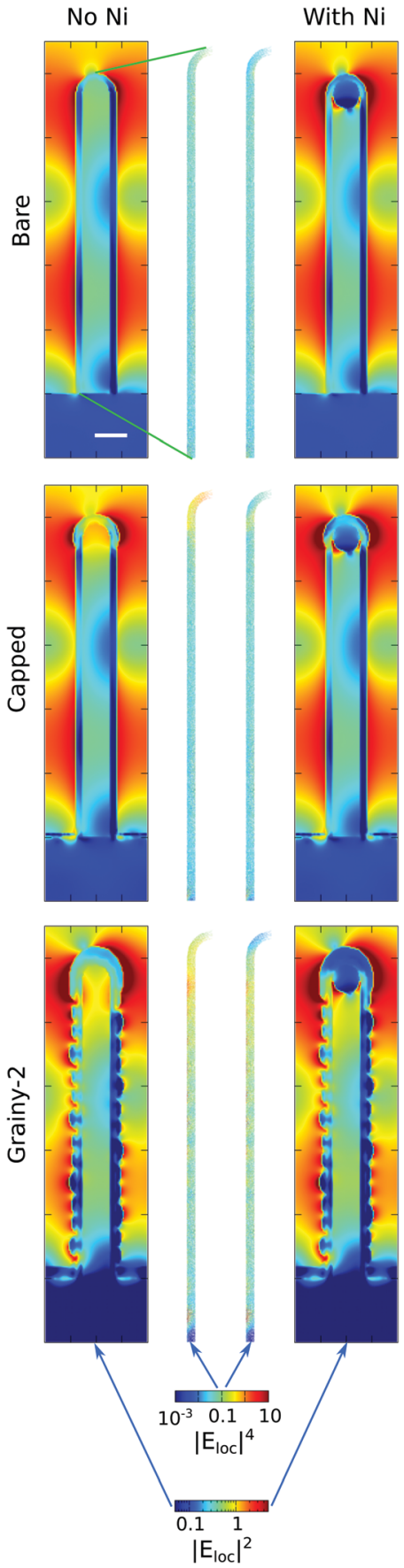

Fig. 7 Images showing variation of $\left|\mathbf{E}_{\mathrm{loc}}\right|^{2}$ and $\left|\mathbf{E}_{\mathrm{loc}}\right|^{4}$, as described previously, for the Bare, Capped and Grainy-2 samples without the Ni nanosphere (left), juxtaposed with those for samples with the Ni nanosphere (right). In all cases p-pol light is incident at $25^{\circ}$ to the MWCNT axis (from top left); scale bar is $50 \mathrm{~nm}$.

that the Bare sample (Fig. 2a) yields a signal from the $\mathrm{Si}$ substrate that is $\sim 50 \times$ greater than that from the $\mathrm{D}$ - and G-bands in terms of peak intensities, but a factor of 0.72 in terms of integrated intensities (the $\mathrm{D}$ - and G-bands are spectrally broad compared to the Si peak) compared to a value of 0.31 in the modelling. However, the model does not take account of differences in the intrinsic Raman cross-sections or changes in the distribution of $\left|\mathbf{E}_{\text {loc }}\right|^{4}$ between incident and scattered wavelengths. In any case, the values of $\left|\mathbf{E}_{\text {loc }}\right|^{4}$ in the first row of Table 1 (normalised to $1 \mathrm{~V} \mathrm{~m}^{-1}$ input) illustrate that the Raman signal originates from a relatively weak concentration of the electromagnetic field within the volume of the MWCNTs.

Addressing first the MWCNT Raman signal it is useful to place our experimental values of EF for Ag-capped and -coated samples (4.2 \pm 0.25 and $14.1 \pm 0.85$ respectively) in the context of related work: $\mathrm{Ag}$ NPs on SWCNTs $(\mathrm{EF}=5,10$ and 12 for excitation wavelengths, $\lambda_{\text {exc }}=785,633$ and $514.5 \mathrm{~nm}$ severally, Chen et $\left.a l .{ }^{30}\right)$; Au NPs on SWCNTs (EF $=8-10$, Sharma et al. $;^{36}$ $\mathrm{EF}=2-3$, Jeong et $\left.a l^{33}\right)$; Ag NPs on MWCNTs $(\mathrm{EF} \sim 10, \mathrm{Lin}$ et $a{ }^{35}{ }^{35}$ ). These $\mathrm{EF}$ values are very much in line with those reported here and indeed also with a report where $\mathrm{Ag}$ core/Au shell NPs on SWCNTs were structurally tuned to match the localised plasmon resonance to $\lambda_{\text {exc }}\left(\mathrm{EF}=9-12, \mathrm{Kumar}^{34}\right)$. To offer further context it is pertinent to note that in tip-enhanced Raman scattering (TERS) where Au tips address CNTs on a dielectric substrate the EF can be as low as 14 (in the range of NP-on-CNT schemes), but up to 770 under optimised conditions. ${ }^{51}$ However, if a SWCNT is sandwiched between an $\mathrm{Ag}$ tip and $\mathrm{Ag}$ substrate in a scanning tunnelling microscope set-up the EF rises to the $\sim 10^{8}$ range $^{52}$ on account of the very large field enhancement driven by localised plasmon modes supported in the gap; ${ }^{41,53,54}$ however, the EF retreats to an estimated $3 \times 10^{4}$ for an Au-tip/MWCNT/Au-substrate tunnel scheme $^{55}$ since the larger diameter of the MWCNT increases the tip-substrate separation, significantly weakening the localised, gap-mode plasmon resonances of the system.

Returning to NP-dressed CNTs some outlier results can help inform us on the disparity between experiment and model in our own data. Large values of EF up to 740 (referenced to a $\mathrm{Si}$ peak) reported by Scolari et al. ${ }^{31}$ were in significant part (almost $50 \times$ ) due to resonant Raman scattering where the absorbed or scattered photon is in resonance with an optical transition between van Hove singularities in the SWCNT; ${ }^{56,57}$ this would imply an electromagnetic EF of $\sim 15$, with perhaps a maximum of $\sim 40$ from other data in that study. Other high EF values were reported by Chu et $a l^{32}$ in an investigation where the Raman enhancement was studied as a function of distance between well-defined spherical Au NPs on SWCNTs; for separations $<10 \mathrm{~nm}$ the EF rose to $\sim 100$.

So why is there a disparity between the experimental and modelled EF in our study? While the modelling indeed produces an enhancement of the MWCNT signal (and an attenuation of the Si signal) with Ag-dressing, the EFs for the MWCNT samples (average of $2.24 \pm 0.21$ for $0^{\circ}$ and $25^{\circ}$ incidence for the Ag-capped sample and $4.10 \pm 0.17$ averaged over samples Grainy-1, -2 and -3 in Table 1) fall below those of the experimental data for both Ag-capped $(\mathrm{EF}=4.22 \pm 0.25)$ and Ag-coated samples $(\mathrm{EF}=14.13 \pm 0.85)$. First, there is likely to be little contribution from a resonance Raman effect since this is expected to be much weaker at any fixed $\lambda_{\text {exc }}$ for MWCNTs on account of the large spread in properties of the constituent nanotubes. Second, the possibility of the so-called 'chemical 
effect' could come into play - the dominance of this effect was used by Lin et $a .^{35}$ to explain an EF of unity, i.e. no Raman enhancement, upon addition of Ag NPs to SWCNTs for $\lambda_{\text {exc }}=$ $532 \mathrm{~nm}$. However, like resonance Raman any such effect is likely to be more pronounced for SWCNTs. ${ }^{56}$ Bearing in mind the higher EF values recorded by Chu et al. ${ }^{32}$ and noted above we therefore consider that most of the discrepancy arises from the highly organised structuring of the modelling not taking into account random hotter regions in the body of the MWCNT induced by the detail of the real $\mathrm{Ag}$ topography e.g. hotspots between asymmetric particles and the precise profiling of the Ag-MWCNT interface.

Turning attention to the Si peak at $521 \mathrm{~cm}^{-1}$ it is seen that the modelling for the cases of Grainy- 2 and -3 is in generally good agreement with experiment. The average AF in the modelled data is $7.3 \pm 1.3$ for Grainy- 2 and $5.9 \pm 0.9$ for Grainy-3 compared to the experimental $\mathrm{AF}=5.77 \pm 0.12$. (On the basis of these data and the general appearance of the modelled structures, Fig. 3, a model structure in the scope of Grainy-2 to Grainy-3 is considered to be the preferred, realistic representation of the physical structures.) The significant discrepancy between the experimental and modelled AF for the Si-peak in the case of Ag-capped structure is thought to be due to rather different optical data for the case of a very thin $\mathrm{Ag}$ film ${ }^{58,59}$ (on the Si substrate). Increased surface and grain boundary scattering relative to thicker films will result in the observed AF value being greater than the modelled value.

The final consideration in the modelling is the inclusion of the Ni particles (spheres) within the hemispherical tops of the MWCNTs. Their effect is illustrated in Fig. 7 for the cases of the Bare, Capped and the Ag-coated, Grainy-2 sample. The primary feature is a concentration of the field intensity $\left|\mathbf{E}_{\text {loc }}\right|^{2}$ in the space between the underside of the $\mathrm{Ni}$ sphere and the inside wall of the MWCNT. This has the effect of decreasing the value of $\left|\mathbf{E}_{\mathrm{loc}}\right|^{4}$ in the hemispherical top portion of the MWCNT itself in both the Capped and Grainy-2 cases. This leads to a significant reduction in the overall EF for the Capped case while that for Grainy-2 actually remains very similar - Table S1 (ESI $\dagger$ ). However, for the Grainy samples (all cases) that assessment comes with the caveat that there is a significant margin of error or spread in $\left|\mathbf{E}_{\text {loc }}\right|^{4}$ values for the p-polarised input at $25^{\circ}$ incident angle. Paradoxically, there are actually increases of $\sim 16 \%$ (s-pol, $0^{\circ}$ ), and $\sim 11 \%$ (p-pol, $25^{\circ}$ ) in the EF in the case of the Coated MWCNT with the Ni inclusion compared to that without, though again it is only the figure for the s-polarised case that may be quoted with a small margin of error $( \pm 4 \%)$ and thus a good degree of confidence. A small increase in EF with the Ni inclusion also carries over to the case of the grainy sample of least rugosity, Grainy-1.

Having addressed the elastic and Raman response of the Ag-dressed MWCNTs in some detail there are several points to note in relation to the spectroscopy itself. The first is a small peak in the spectra at $\sim 1150 \mathrm{~cm}^{-1}$ that is observed only in the case of the Ag-dressed MWCNTs; it is most distinct for the Ag-capped and fully-coated samples (Fig. $2 b$ and e). One possibility is that it is related to the $\mathrm{Ag}$ but it would not appear that there is any common $\mathrm{Ag}$ compound or corrosion product involving oxygen, environmental sulfur or carbon that generates a Raman peak in the $1100-1200 \mathrm{~cm}^{-1}$ range. ${ }^{60,61}$ We therefore consider that the principal candidate from the literature on carbon material synthesis to be trans-polyacetylene (TPA), an organic polymer with the repeating unit $\left(\mathrm{C}_{2} \mathrm{H}_{2}\right)_{n}$. A material of some interest in its own right, first examined in detail by Raman spectroscopy in the 1980 's, ${ }^{62-64}$ its occurrence in the Raman spectra of synthesised carbon materials was first noted in the context of chemical-vapour-deposited (CVD) nano-crystalline diamond films. ${ }^{65}$ Both prior to, and after this identification the Raman peak at $\sim 1150 \mathrm{~cm}^{-1}$ (along with an accompanying peak at $\sim 1450 \mathrm{~cm}^{-1}$ ) was generally incorrectly attributed to nano-crystalline diamond itself. ${ }^{66,67}$ However, Ferrari and Robertson $^{68}$ argued on multiple grounds, appealing to previously developed theoretical background, ${ }^{64}$ that these spectral features could not have their origin with $\mathrm{sp}^{3}$ bonding, but instead pointed decisively to the presence of TPA, associated with grain boundary regions in the nano-crystalline structure. The peak at $\sim 1450 \mathrm{~cm}^{-1}$ is associated with the $\mathrm{C}=\mathrm{C}$ stretching vibration while that at $\sim 1150 \mathrm{~cm}^{-1}$ with in-plane $\mathrm{C}-\mathrm{H}$ bending (coupled to the $\mathrm{C}=\mathrm{C}$ stretching mode). Subsequent experiments involving Raman spectroscopy of variously prepared and treated CVD diamond films, including deuterated films, confirmed TPA as the origin of the Raman peaks under discussion. ${ }^{69}$ Thus far, little has been reported on TPA in the context of carbon nanotubes. A significant exception, however, is a report on the formation of TPA on laser irradiated CNT samples which had adsorbed water vapour from the atmosphere. ${ }^{70}$ It is also interesting to note that modelling of the adsorption of $\mathrm{C}_{2} \mathrm{H}_{2}$ on carbon-doped boron nitride nanotubes using density-functional theory has identified the formation of TPA as energetically favourable. $^{71}$ In conclusion, we suggest that the peak at $1150 \mathrm{~cm}^{-1}$ (with also arguably a small shoulder around $\sim 1450 \mathrm{~cm}^{-1}$ in the most enhanced spectra, i.e. where the accompanying $\mathrm{C}=\mathrm{C}$ stretch peak would be expected) is due to TPA and that it is possibly associated primarily with the cap region of the MWCNTs in the vicinity of the Ni catalyst NP. Note from Fig. 4c that the Ag-capped sample exhibits the greatest Raman enhancement of all the samples in this region and it is for this sample that the $1150 \mathrm{~cm}^{-1}$ peak stands out most from the tail of the D-band background (Fig. 2b); there is also a reasonably enhanced value of $\left|\mathbf{E}_{\mathrm{loc}}\right|^{4}$ in this region of the MWCNTs for the various Ag-coated samples (Fig. 4c), where again the $1150 \mathrm{~cm}^{-1}$ peak is present in the Raman spectra. It seems most likely that TPA formation would occur at the Ni NP catalyst either during synthesis $-\mathrm{C}_{2} \mathrm{H}_{2}$ is the active precursor gas ${ }^{43,44}$ - or possibly in the course of the Raman spectral acquisition ${ }^{70}$ i.e. stimulated by laser irradiation in the presence of adsorbed water. However, the latter route seems less likely here since the MWCNT samples were stored in a vacuum-desiccator prior to $\mathrm{Ag}$ deposition and examination. Since the $1150 \mathrm{~cm}^{-1}$ peak is very small and the Raman cross-section of TPA is very large it would be present in only very small quantities. ${ }^{68}$

The second point in relation to the spectroscopy is a slight increase in the disorder-induced D-band peak relative to the 


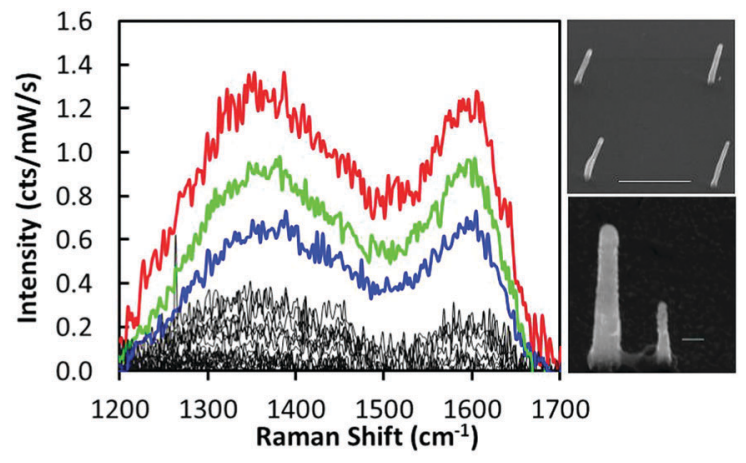

Fig. 8 Spectra from sample with MWCNTs grown in a square array of period $1.8 \mu \mathrm{m}$ and dressed with granular Ag coating of nominal thickness $24 \mathrm{~nm}$, as shown in SEM images where scale bars are $1 \mu \mathrm{m}$ (top) and $100 \mathrm{~nm}$ (bottom). Spectra were acquired during step-wise scanning. Black spectra principally sample regions between MWCNTs and coloured spectra regions in which MWCNT is at or near centre of the focused input laser beam. A $50 \times$ objective was used in the Raman microscope, yielding laser spot size of $\sim 2 \mu \mathrm{m}$ diameter; $\lambda=632.8 \mathrm{~nm}, P_{\text {in }}=5 \mathrm{~mW}$; acquisition performed in $5 \times 10$ s captures.

G-band with Ag dressing. The ratio of D- to G-band intensity, $I_{\mathrm{D}} / I_{\mathrm{G}}$, increases from approximately unity for the bare nanotubes to a value in the range 1.10-1.15 for both the Ag-capped and fully Ag-coated nanotubes. This feature has been noted and discussed in some detail by Zhang et al. ${ }^{26}$ A pertinent connection can be made to the TERS investigation of Liao et al. ${ }^{52}$ who showed in separate Raman maps of the D- and G-bands of single CNTs that the bulk of the D-band signal originates from the top of CNTs, with the G-band intensity being distributed much more evenly along the length of the nanotube. Given the local concentration of $\left|\mathbf{E}_{\text {loc }}\right|^{4}$ near the top of the Ag-capped and -coated MWCNTs relative to their bare counterparts it seems reasonable to suggest that most of the increase in $I_{\mathrm{D}} / I_{\mathrm{G}}$ upon Ag dressing is due to increased D-band signal from the tops of the MWCNTs.

Lastly and importantly, the spectra from individual, Ag-coated MWCNTs (Fig. 8) are very similar in structure to those averaged over multiple MWCNTs (Fig. 2) with broad D- and G-bands, the D-band being slightly stronger, as noted above. This is clear evidence that the structural imperfections underlying such spectra are a characteristic of the individual nanotubes and not an ensemble average of MWCNTs of differing spectral content. In Fig. 8 the spectra shown were taken in scanning the surface of samples in which the MWCNTs were grown on a square grid of period $\sim 1.8 \mu \mathrm{m}$. It is only when the probe laser beam is incident approximately centrally on a MWCNT that a discernible spectrum is retrieved and, notably, only when the MWCNT is dressed with $\mathrm{Ag}$ - no such spectra are acquired from the as-prepared bare sample under the same collection conditions.

\section{Conclusions}

When random arrays of vertically aligned MWCNTs (of average length $\sim 500 \mathrm{~nm}$ ) are dressed with nano-particulate $\mathrm{Ag}$ it is found that the D- and G-band Raman signals from the nanotubes are enhanced while, simultaneously, the signal from the Si substrate is attenuated. The respective enhancement and attenuation factors are up to $\sim 14 \times$ and $\sim 6 \times$ respectively, giving a discrimination of almost 2 orders of magnitude in favour of the MWCNT Raman signature over that of the Si substrate, compared to the native MWCNT-on-Si system. In the present experimental set-up Ag dressing has facilitated the ready characterisation of individual MWCNTs - grown on a square grid pattern of period $1.8 \mu \mathrm{m}$ - that would otherwise have been very time-consuming to recover, showing that the broad D and G-bands are characteristic of individual nanotubes and are not representative of an ensemble average. Specifically, the defect-related D-band is an intrinsic characteristic of individual MWCNTs. Moreover, it is only for the Ag-dressed samples that a small peak shows up at $\sim 1150 \mathrm{~cm}^{-1}$ which we attribute to the presence of trans-polyacetylene, probably in the vicinity of the Ni catalyst NP at the top of the MWCNT.

Detailed modelling of the electromagnetic response has been carried out using the SIE technique; in addition to the elastic optical response of the system as depicted in 2D images of $\left|\mathbf{E}_{\text {loc }}(\omega)\right|^{2}$ the Raman response has been estimated by evaluating $\left|\mathbf{E}_{\mathrm{loc}}(\omega)\right|^{4}$ within the volume of the MWCNT, modelled with a wall thickness of $10 \mathrm{~nm}$. Although quantitative agreement with experiment is not achieved, valuable insights are gained on the underlying enhancement mechanism. In the case of Ag-capped samples much of the MWCNT Raman enhancement comes from the hemispherical cap region. The overall antenna response of the MWCNTs, present in the Bare case, but amplified by coating with nano-particulate Ag, accounts for the majority of the Raman response for samples designated as Grainy in the modelling. Regions of intense electric field confinement between adjacent Ag NPs on the surface of the MWCNTs are very well confined between the NPs themselves and penetrate the MWCNTs only to a limited degree - hence our choice of terminology of warm-spots, rather than hot-spots. The warm-spots within the MWCNTs are shown to be in direct spatial correspondence with the hot-spots between Ag NPs and to be most pronounced for the case of p-polarised light at non-normal incidence ( $25^{\circ}$ in the modelling) where there is a component of the input electric field linking the NPs along the axial direction of the MWCNTs. (In the orthogonal, s-polarised case the curvature of the MWCNT means that nearestneighbour Ag NPs lie in quite differently oriented vertical planes, yielding much weaker coupling between them.) The efficacy of the antenna response in producing Raman enhancement is underlined by the case of MWCNTs with a conformal Ag coating in the modelling (but not replicated experimentally here). The discrepancy between EFs in experiment and model is not thought to be due to resonance Raman scattering or the 'chemical effect' but more likely an underestimation of the role of random warm-spots not captured in the highly symmetrical model structures.

In summary, and to place the present investigation in the context of our previous related work, ${ }^{7}$ for Ag NP-coated MWCNTs there is significant contrast between the Raman enhancement mechanism of the MWCNTs themselves and that driving the markedly more enhanced Raman signal from 
molecules adsorbed onto this framework structure. In the former case, the antenna response makes a key contribution but plays just a background role in the molecular adsorbate case. For molecules adsorbed on the Ag-NPs the hot-spots between adjacent Ag-NPs are crucial to the Raman EF, as are the hotspots between co-leaning or 'kissing' Ag-coated MWCNTs;' this is a heavily hot-spot dominated system. In contrast, it is re-iterated that the inter-Ag particle hotspots make a more limited contribution to the Raman enhancement of MWCNTs, manifesting only as 'warm' spots within the MWCNT volume itself. There is no contribution from interMWCNT hot-spots since such hotspots are generated by capillary forces during the drying of solvent deposited on the surface, used to deposit molecular adsorbates, ${ }^{21}$ i.e. there are no co-leaning or 'kissing' nanotubes (see insets to Fig. 1a, 2a and d).

Finally, and more broadly, either conformal, thin-film or nano-particulate dressings of a plasmonic metal may be deployed on a variety of nanopillar structures to facilitate enhanced Raman analysis of the structures (individually or collectively in arrays), while simultaneously offering significant attenuation of background signals from the substrate. The procedure could be applied to various semiconductor nanowires/nanopillars ${ }^{72-74}$ where, moreover, such configurations offer a route to the realisation of high-aspect ratio Schottky diodes with the dual features of an enhanced, plasmonically resonant antenna response ${ }^{75}$ and the possibility of using an alternative, simple 2-contact configuration for sensing applications. ${ }^{76,77}$

\section{Methods}

The vertically aligned MWCNTs were grown using plasma enhanced chemical vapour deposition (PECVD). The process first involves the formation of $\mathrm{Ni}$ catalyst islands on a $\mathrm{Si}$ substrate by sintering a thin Ni film $\left(\sim 5 \mathrm{~nm}\right.$ thick) at $700{ }^{\circ} \mathrm{C}$. Nanotube growth proceeds from these discrete island catalysts resulting in highly aligned, untangled MWCNTs (see Fig. 1a, inset). The nanotube growth proceeds on the underside of the $\mathrm{Ni}$ such that the Ni particle resides at the top of the nanotube the MWCNT-encased Ni NPs are clearly visible in the inset SEM image of Fig. 1a. The growth itself proceeds in a DC plasma discharge (bias $-600 \mathrm{~V}$ ) of acetylene and ammonia using flow rates of $100-200 \mathrm{sccm}$ at a partial pressure of 4-5 Torr. The MWCNT diameter and areal density are determined by the thickness of the Ni film while the nanotube height is a function of the plasma deposition time. Following an initial growth phase, a rate of $\sim 300 \mathrm{~nm}$ per minute is typically established. The most explicit descriptions of the growth are given in the early papers on the PECVD method, ${ }^{43,44}$ including analysis of the role of the plasma environment, ${ }^{78,79}$ the metal catalyst composition $^{80}$ and the elimination of amorphous carbon from the MWCNT samples; ${ }^{43,44,81}$ much of this work was driven by applications such as field emitter devices ${ }^{82}$ and superhydrophobic surfaces. ${ }^{83}$ The Ag dressing of the MWCNT forests was performed in a thermal evaporator (Edwards 306 series) at a base pressure of $3.0-5.0 \times 10^{-6}$ Torr and deposition rate of $\sim 0.5 \mathrm{~nm} \mathrm{~s}^{-1}$. The Ag-capped sample was produced by $\mathrm{Ag}$ deposition that was performed as close to normal incidence to the substrate as possible by use of a small basket source, substrate masking (with the mask adjacent the substrate surface of $\sim 1.2 \mathrm{~mm}$ aperture) and maximising the source-substrate distance. The Ag coating of the MWCNT was performed by spinning the MWCNT substrate at a rate of $\sim 1 \mathrm{~Hz}$ with the substrate normal at an angle of $\sim 15^{\circ}$ to the direction of the incident evaporant. The Raman spectra were recorded with an input laser wavelength of $632.8 \mathrm{~nm}$ using a Jobin-Yvon LabRam microscope equipped with $50 \times$ objective (numerical aperture 0.55 ). The exposure conditions are given in the captions to Fig. 2 and 8.

Modelling the electromagnetic response of the various nanotube structures was done using a 3D surface integral equation (SIE) technique as described by Kern and Martin, ${ }^{42}$ developed by Gallinet et $a l^{84}$ for application to periodic nanostructures and applied in our previous analysis of Ag-dressed carbon nanotube arrays. ${ }^{7}$ The method is well suited to the simulation of plasmonic and other optical resonance phenomena occurring in complex geometrical structures on account of its computational efficiency. This derives from the fact that the technique requires the discretization of only the boundaries of the scattering entities and not their volumes or the background as well; the various meshes used here are illustrated in Fig. 3. Relative to the earlier work ${ }^{42,84}$ the principal development in the SIE technique as applied here is an optimized integral evaluation method. ${ }^{85}$ Once the surface currents and local fields have been calculated it is required to evaluate the Raman signal that arises throughout the volume of the MWCNT walls and the Si substrate. In order to compute the average Raman signal over each domain a Monte Carlo approach was adopted in which 40000 points were chosen uniformly randomly in the volume of the nanotube walls, and similarly in the Si substrate. Since the geometry of the nanotube is complicated and the mesh is too discrete to be specified by mathematical formulae, it is ensured that the points are inside the correct domain by the implementation of binary space partitioning. ${ }^{86}$ This procedure is not required for the Si substrate since the boundary is flat; the semi-infinite substrate is truncated at a depth of $10 \mu \mathrm{m}$ for Monte Carlo point sampling and averaging. Ideally, the Raman signal should be calculated by finding the field intensity enhancement at each point and then using this to compute the radiated far field. ${ }^{87,88}$ However, this would require treating each point in the Monte Carlo sampling as a separate illumination for SIE, making this treatment prohibitively expensive. We hence make the approximation that the Raman signal from a point is proportional to $\left|\mathbf{E}_{\text {loc }}(\omega)\right|^{4}$ where $\mathbf{E}_{\text {loc }}(\omega)$ is the local electric field at the illumination frequency (wavelength).

\section{Conflicts of interest}

There are no conflicts to declare.

\section{Acknowledgements}

PD gratefully acknowledges support from the Department for the Economy, Northern Ireland (Project USI-043) and the EC COST program; OJFM and TVR acknowledge support from the 
European Research Council (ERC-2015-AdG-695206 Nanofactory) and the Swiss National Science Foundation (Project 200020_135452). PD also thanks Michael Boyle and Mike Hardy (Centre for Nanostructured Media, QUB) for assistance with data analysis and calibration measurements and Stephen Bell (Dept. of Chemistry, QUB) for use of the Raman microscope.

\section{References}

1 M. Moskovits, J. Raman Spectrosc., 2005, 36, 485-496.

2 W. H. Zhang, H. Fischer, T. Schmid, R. Zenobi and O. J. F. Martin, J. Phys. Chem. C, 2009, 113, 14672-14675.

3 Y. J. Oh, M. Kang, M. Park and K. H. Jeong, BioChip J., 2016, 10, 297-309.

4 Y. Fang, N. H. Seong and D. D. Dlott, Science, 2008, 321, 388-392.

5 M. D. Doherty, A. Murphy, J. McPhillips, R. J. Pollard and P. Dawson, J. Phys. Chem. C, 2010, 114, 19913-19919.

6 M. D. Doherty, A. Murphy, R. J. Pollard and P. Dawson, Phys. Rev. X, 2013, 3, 011001.

7 P. Dawson, J. A. Duenas, M. G. Boyle, M. D. Doherty, S. E. J. Bell, A. M. Kern, O. J. F. Martin, A. S. Teh, K. B. K. Teo and W. I. Milne, Nano Lett., 2011, 11, 365-371. 8 M. Fleischmann, P. J. Hendra and A. J. McQuillan, Chem. Phys. Lett., 1974, 26, 163-166.

9 G. Amarandei, C. O'Dwyer, A. Arshak and D. Corcoran, ACS Appl. Mater. Interfaces, 2013, 5, 8655-8662.

10 A. Campion and P. Kambhampati, Chem. Soc. Rev., 1998, 27, 241-250.

11 Z. Wang, S. Pan, T. D. Krauss, H. Du and L. J. Rothberg, Proc. Natl. Acad. Sci. U. S. A., 2003, 100, 8638-8643.

12 S. Botti, S. Almaviva, L. Cantarini, A. Palucci, A. Puiu and A. Rufoloni, J. Raman Spectrosc., 2013, 44, 463-468.

13 S. Botti, L. Cantarini, S. Almaviva, A. Puiu and A. Rufoloni, Chem. Phys. Lett., 2014, 592, 277-281.

14 M. Hardy, M. D. Doherty, I. Krstev, K. Maier, T. Moller, G. Muller and P. Dawson, Anal. Chem., 2014, 86, 9006-9012.

15 S. B. Chaney, S. Shanmukh, R. A. Dluhy and Y. P. Zhao, Appl. Phys. Lett., 2005, 87, 031908.

16 A. Ahmed and R. Gordon, Nano Lett., 2012, 12, 2625-2630.

17 Y. T. Kim, J. Schilling, S. L. Schweizer and R. B. Wehrspohn, Plasmonics, 2017, 12, 203-208.

18 Z. W. Zuo, K. Zhu, L. X. Ning, G. L. Cui, J. Qu, Y. Cheng, J. Z. Wang, Y. Shi, D. S. Xu and Y. Xin, Appl. Surf. Sci., 2015, 325, 45-51.

19 W. Wu, M. Hu, F. S. Ou, Z. Y. Li and R. S. Williams, Nanotechnology, 2010, 21, 255502.

20 K. Wu, T. Rindzevicius, M. S. Schmidt, K. B. Mogensen, S. Xiao and A. Boisen, Opt. Express, 2015, 23, 12965-12978.

21 M. S. Schmidt, J. Hubner and A. Boisen, Adv. Mater., 2012, 24, OP11-OP18.

22 Y. J. Oh and K. H. Jeong, Adv. Mater., 2012, 24, 2234-2237.

23 P. Lovera, N. Creedon, H. Alatawi, M. Mitchell, M. Burke, A. J. Quinn and A. O'Riordan, Nanotechnology, 2014, 25, 175502 .
24 A. O. Altun, S. K. Youn, N. Yazdani, T. Bond and H. G. Park, Adv. Mater., 2013, 25, 4431-4436.

25 J. Zhang, T. Fan, X. Zhang, C. Lai and Y. Zhu, Appl. Opt., 2014, 53, 1159-1165.

26 J. Zhang, X. Zhang, S. Chen, T. Gong and Y. Zhu, Carbon, 2016, 100, 395-407.

27 X. Zhang, J. Zhang, J. Quan, N. Wang and Y. Zhu, Analyst, 2016, 141, 5527-5534.

28 P. Goldberg-Oppenheimer, T. Hutter, B. Chen, J. Robertson, S. Hofmann and S. Mahajan, J. Phys. Chem. Lett., 2012, 3, 3486-3492.

29 X. Wang, C. Wang, L. Cheng, S.-T. Lee and Z. Liu, J. Am. Chem. Soc., 2012, 134, 7414-7422.

30 Y. C. Chen, R. J. Young, J. V. Macpherson and N. R. Wilson, J. Phys. Chem. C, 2007, 111, 16167-16173.

31 M. Scolari, A. Mews, N. Fu, A. Myalitsin, T. Assmus, K. Balasubramanian, M. Burghard and K. Kern, J. Phys. Chem. C, 2008, 112, 391-396.

32 H. B. Chu, J. Y. Wang, L. Ding, D. N. Yuan, Y. Zhang, J. Liu and Y. Li, J. Am. Chem. Soc., 2009, 131, 14310-14316.

33 G. H. Jeong, S. Suzuki and Y. Kobayashi, Nanotechnology, 2009, 20, 285708.

34 G. V. P. Kumar, J. Raman Spectrosc., 2009, 40, 2069-2073.

35 Y. Lin, K. A. Watson, M. J. Fallbach, S. Ghose, J. G. Smith, D. M. Delozier, W. Cao, R. E. Crooks and J. W. Connell, ACS Nano, 2009, 3, 871-884.

36 H. Sharma, D. C. Agarwal, A. K. Shukla, D. K. Avasthi and V. D. Vankar, J. Raman Spectrosc., 2013, 44, 12-20.

37 H. Im, K. C. Bantz, N. C. Lindquist, C. L. Haynes and S. H. Oh, Nano Lett., 2010, 10, 2231-2236.

38 H. G. Duan, H. L. Hu, K. Kumar, Z. X. Shen and J. K. W. Yang, ACS Nano, 2011, 5, 7593-7600.

39 Q. Fu, Z. B. Zhan, J. X. Dou, X. Z. Zheng, R. Xu, M. H. Wu and Y. Lei, ACS Appl. Mater. Interfaces, 2015, 7, 13322-13328.

40 H. B. Cai, Y. K. Wu, Y. M. Dai, N. Pan, Y. C. Tian, Y. Luo and X. P. Wang, Opt. Express, 2016, 24, 20808-20815.

41 P. Dawson, D. Frey, V. Kalathingal, R. Mehfuz and J. Mitra, Faraday Discuss., 2017, 205, 121-148.

42 A. M. Kern and O. J. F. Martin, J. Opt. Soc. Am. A, 2009, 26, 732-740.

43 M. Chhowalla, K. B. K. Teo, C. Ducati, N. L. Rupesinghe, G. A. J. Amaratunga, A. C. Ferrari, D. Roy, J. Robertson and W. I. Milne, J. Appl. Phys., 2001, 90, 5308-5317.

44 K. B. K. Teo, M. Chhowalla, G. A. J. Amaratunga, W. I. Milne, D. G. Hasko, G. Pirio, P. Legagneux, F. Wyczisk and D. Pribat, Appl. Phys. Lett., 2001, 79, 1534-1536.

45 T. L. Sun, H. A. Liu, W. L. Song, X. Wang, L. Jiang, L. Li and D. B. Zhu, Angew. Chem., Int. Ed., 2004, 43, 4663-4666.

46 M. G. Donato, S. Galvagno, G. Messina, C. Milone, A. Pistone and S. Santangelo, Diamond Relat. Mater., 2007, 16, 1095-1100.

47 P. Sampedro-Tejedor, A. Maroto-Valiente, D. M. Nevskaia, V. Munoz, I. Rodriguez-Ramos and A. Guerrero-Ruiz, Diamond Relat. Mater., 2007, 16, 542-549.

48 P. Alonso-Gonzalez, P. Albella, M. Schnell, J. Chen, F. Huth, A. Garcia-Etxarri, F. Casanova, F. Golmar, L. Arzubiaga, 
L. E. Hueso, J. Aizpurua and R. Hillenbrand, Nat. Commun., 2012, 3, 684 .

49 M. Bruna and S. Borini, Appl. Phys. Lett., 2009, 94, 031901.

50 L. Novotny, Phys. Rev. Lett., 2007, 98, 266802.

51 L. G. Cancado, A. Hartschuh and L. Novotny, J. Raman Spectrosc., 2009, 40, 1420-1426.

52 M. H. Liao, S. Jiang, C. R. Hu, R. Zhang, Y. M. Kuang, J. Z. Zhu, Y. Zhang and Z. C. Dong, Nano Lett., 2016, 16, 4040-4046.

53 J. Mitra, L. Feng, M. G. Boyle and P. Dawson, J. Phys. D: Appl. Phys., 2009, 42, 215101.

54 V. Kalathingal, P. Dawson and J. Mitra, Phys. Rev. B: Condens. Matter Mater. Phys., 2016, 94, 035443.

55 G. Picardi, M. Chaigneau and R. Ossikovski, Chem. Phys. Lett., 2009, 469, 161-165.

56 P. Corio, S. D. M. Brown, A. Marucci, M. A. Pimenta, K. Kneipp, G. Dresselhaus and M. S. Dresselhaus, Phys. Rev. B: Condens. Matter Mater. Phys., 2000, 61, 13202-13211.

57 A. Jorio, M. A. Pimenta, A. G. Souza, R. Saito, G. Dresselhaus and M. S. Dresselhaus, New J. Phys., 2003, 5, 139.

58 T. W. H. Oates, L. Ryves, M. M. M. Bilek and D. R. McKenzie, Sens. Actuators, B, 2005, 109, 146-152.

59 T. W. H. Oates, L. Ryves and M. M. M. Bilek, Opt. Express, 2008, 16, 2302-2314.

60 I. Martina, R. Wiesinger and M. Schreiner, J. Raman Spectrosc., 2013, 44, 770-775.

61 R. Wiesinger, I. Martina, C. Kleber and M. Schreiner, Corros. Sci., 2013, 77, 69-76.

62 I. Harada, Y. Furukawa, M. Tasumi, H. Shirakawa and S. Ikeda, J. Chem. Phys., 1980, 73, 4746-4757.

63 L. S. Lichtmann, A. Sarhangi and D. B. Fitchen, Solid State Commun., 1980, 36, 869-873.

64 E. Ehrenfreund, Z. Vardeny, O. Brafman and B. Horovitz, Phys. Rev. B: Condens. Matter Mater. Phys., 1987, 36, 1535-1553.

65 T. LopezRios, E. Sandre, S. Leclercq and E. Sauvain, Phys. Rev. Lett., 1996, 76, 4935-4938.

66 R. J. Nemanich, J. T. Glass, G. Lucovsky and R. E. Shroder, J. Vac. Sci. Technol., A, 1988, 6, 1783-1787.

67 K. Okada, H. Kanda, S. Komatsu and S. Matsumoto, J. Appl. Phys., 2000, 88, 1674-1678.

68 A. C. Ferrari and J. Robertson, Phys. Rev. B: Condens. Matter Mater. Phys., 2001, 63, 121405.

69 H. Kuzmany, R. Pfeiffer, N. Salk and B. Gunther, Carbon, 2004, 42, 911-917.

70 M. Hakamatsuka, H. Yoshimura and M. Tachibana, Carbon, 2011, 49, 1869-1872.
71 Y. Chen, H. X. Wang, J. X. Zhao, Q. H. Cai, X. G. Wang and X. Z. Wang, J. Mol. Model., 2012, 18, 3415-3425.

72 R. Ghosh and P. K. Giri, RSC Adv., 2016, 6, 35365-35377.

73 B. Ketterer, M. Heiss, M. J. Livrozet, A. Rudolph, E. Reiger and A. F. I. Morral, Phys. Rev. B: Condens. Matter Mater. Phys., 2011, 83, 125307.

74 S. A. Razek, M. A. Swillam and N. K. Allam, J. Appl. Phys., 2014, 115, 194305.

75 Z. H. Chen, Y. B. Tang, C. P. Liu, Y. H. Leung, G. D. Yuan, L. M. Chen, Y. Q. Wang, I. Bello, J. A. Zapien, W. J. Zhang, C. S. Lee and S. T. Lee, J. Phys. Chem. C, 2009, 113, 13433-13437.

76 L. Feng, J. Mitra, P. Dawson and G. Hill, J. Phys.: Condens. Matter, 2011, 23, 422201.

77 J. Mitra, L. Feng, L. Penate-Quesada and P. Dawson, J. Appl. Phys., 2015, 117, 244501.

78 D. B. Hash, M. S. Bell, K. B. K. Teo, B. A. Cruden, W. I. Milne and M. Meyyappan, Nanotechnology, 2005, 16, 925-930.

79 K. B. K. Teo, D. B. Hash, R. G. Lacerda, N. L. Rupesinghe, M. S. Bell, S. H. Dalal, D. Bose, T. R. Govindan, B. A. Cruden, M. Chhowalla, G. A. J. Amaratunga, J. M. Meyyappan and W. I. Milne, Nano Lett., 2004, 4, 921-926.

80 R. G. Lacerda, K. B. K. Teo, A. S. Teh, M. H. Yang, S. H. Dalal, D. A. Jefferson, J. H. Durrell, N. L. Rupesinghe, D. Roy, G. A. J. Amaratunga, W. I. Milne, F. Wyczisk, P. Legagneux and M. Chhowalla, J. Appl. Phys., 2004, 96, 4456-4462.

81 R. G. Lacerda, A. S. Teh, M. H. Yang, K. B. K. Teo, N. L. Rupesinghe, S. H. Dalal, K. K. K. Koziol, D. Roy, G. A. J. Amaratunga, W. I. Milne, M. Chhowalla, D. G. Hasko, F. Wyczisk and P. Legagneux, Appl. Phys. Lett., 2004, 84, 269-271.

82 W. I. Milne, K. B. K. Teo, G. A. J. Amaratunga, P. Legagneux, L. Gangloff, J. P. Schnell, V. Semet, V. T. Binh and O. Groening, J. Mater. Chem., 2004, 14, 933-943.

83 K. K. S. Lau, J. Bico, K. B. K. Teo, M. Chhowalla, G. A. J. Amaratunga, W. I. Milne, G. H. McKinley and K. K. Gleason, Nano Lett., 2003, 3, 1701-1705.

84 B. Gallinet, A. M. Kern and O. J. F. Martin, J. Opt. Soc. Am. A, 2010, 27, 2261-2271.

85 T. V. Raziman, W. R. C. Somerville, O. J. F. Martin and E. C. Le Ru, J. Opt. Soc. Am. B, 2015, 32, 485-492.

86 M. S. Paterson and F. F. Yao, Discrete Comput. Geom., 1990, 5, 485-503.

87 A. M. Kern, A. J. Meixner and O. J. F. Martin, ACS Nano, 2012, 6, 9828-9836.

88 D. A. Weitz, S. Garoff, J. I. Gersten and A. Nitzan, J. Chem. Phys., 1983, 78, 5324-5338. 